\title{
ANÁLISE QUANTITATIVA DAS ASSOCIAÇÕES DE FORAMINÍFEROS DA PASSAGEM JURÁSSICO INFERIOR-MÉDIO DO PERFIL DE MARIA PARES (SETOR NORTE DA BACIA LUSITÂNICA, PORTUGAL) - IMPLICAÇÕES PALEOECOLÓGICAS
}

\author{
QUANTITATIVE ANALYSIS OF THE LOWER-MIDDLE JURASSIC \\ FORAMINIFERAL ASSEMBLAGES FROM THE MARIA PARES SECTION \\ (NORTHERN LUSITANIAN BASIN, PORTUGAL) - PALEOECOLOGICAL \\ IMPLICATIONS
}

\section{L. FIGUEIREDO ${ }^{(1)}$ e H. C. GUTERRES ${ }^{(2)}$}

\begin{abstract}
Resumo - As associações de foraminíferos bentónicos do intervalo estratigráfico Toarciano superior - Aaleniano médio do Perfil de Maria Pares (Setor Norte da Bacia Lusitânica, Portugal) são características do domínio boreal e típicas de plataformas carbonatadas do Jurássico. A análise quantitativa daquelas permitiu avaliar a sua diversidade, bem como a sua evolução ao longo do intervalo estratigráfico estudado. A partir da análise dos valores obtidos, decorrentes da aplicação de vários índices de diversidade, é possível concluir que as associações estudadas são relativamente diversas e ocupariam zonas de plataforma marinha, com salinidade normal e bem oxigenadas. Além disso, foi também possível diferenciar, ao longo do intervalo estratigráfico estudado, três episódios nos quais as condições ambientais condicionaram o desenvolvimento das associações de foraminíferos.
\end{abstract}

Palavras-chave - Foraminíferos, Paleoecologia, passagem Jurássico Inferior-Médio, Bacia Lusitânica, Portugal.

Abstract - The benthonic foraminiferal assemblages of the upper Toarcian-middle Aalenian from the Maria Pares Section (Northern Sector Lusitanian Basin, Portugal) are characteristic of the Boreal realm and are typical of Jurassic carbonate platforms. The quantitative analysis of the foraminiferal assemblages allowed the evaluation of their diversity, as well as their evolution throughout the studied stratigraphic interval. The resulting values, based on the application of several diversity indexes, indicate that the studied assemblages are relatively diverse, and must have occupied areas of shelfal basin, displaying well-oxygenated and normal salinity conditions. Additionally, it was also possible to differentiate three stages

(1) Laboratório Nacional de Energia e Geologia. Estrada da Portela, Bairro do Zambujal - Alfragide, 2610-999 Amadora. Centro Geociências UC. Lg. Mq. Pombal, 3049 Coimbra, Portugal; verfigueiredo@ gmail.com

(2) Secretaria de Estado de Recursos Naturais. $1^{\circ}$ Andar Edifício de Fomento, Rua Dom Alexo Corte Real, Mandarin, P. O. BOX 171 Dili, Timor Leste. Centro Geociências UC. Lg. Mq. Pombal, 3049 Coimbra, Portugal; larimata@gmail.com 
along the considered time interval, reflecting environmental conditions which have allowed the development of the foraminiferal assemblages.

Keywords - Foraminifers, Paleoecology, Lower-Middle Jurassic boundary, Lusitanian Basin, Portugal.

\section{1 - INTRODUÇÃO}

No presente trabalho apresentam-se os resultados obtidos a partir da análise quantitativa detalhada de 19 associações de foraminíferos bentónicos registadas ao longo do intervalo estratigráfico Toarciano superior (Biozona Aalensis) - Aaleniano inferior (Biozona Opalinum) - Aaleniano médio (Biozona Bradfordensis) no Perfil Maria Pares (Sector Norte da Bacia Lusitânica, Portugal).

A análise quantitativa das associações de foraminíferos foi realizada através da aplicação de vários índices de diversidade. A análise dos valores obtidos permitiu avaliar a diversidade de cada associação, bem como a sua evolução ao longo do intervalo estratigráfico estudado. Foi igualmente possível, para o mesmo intervalo temporal, identificar as condições paleoambientais em que se desenvolveram as associações de foraminíferos e diferenciar alguns episódios onde se registaram variações nas condições ambientais.

\section{2 - ENQUADRAMENTO GEOLÓGICO}

O Perfil de Maria Pares localiza-se no Setor Norte da Bacia Lusitânica (Portugal), na região de Rabaçal, a cerca de 8 km a SE de Condeixa-a-Nova (GUTERRES, 2010) (Fig. 1).

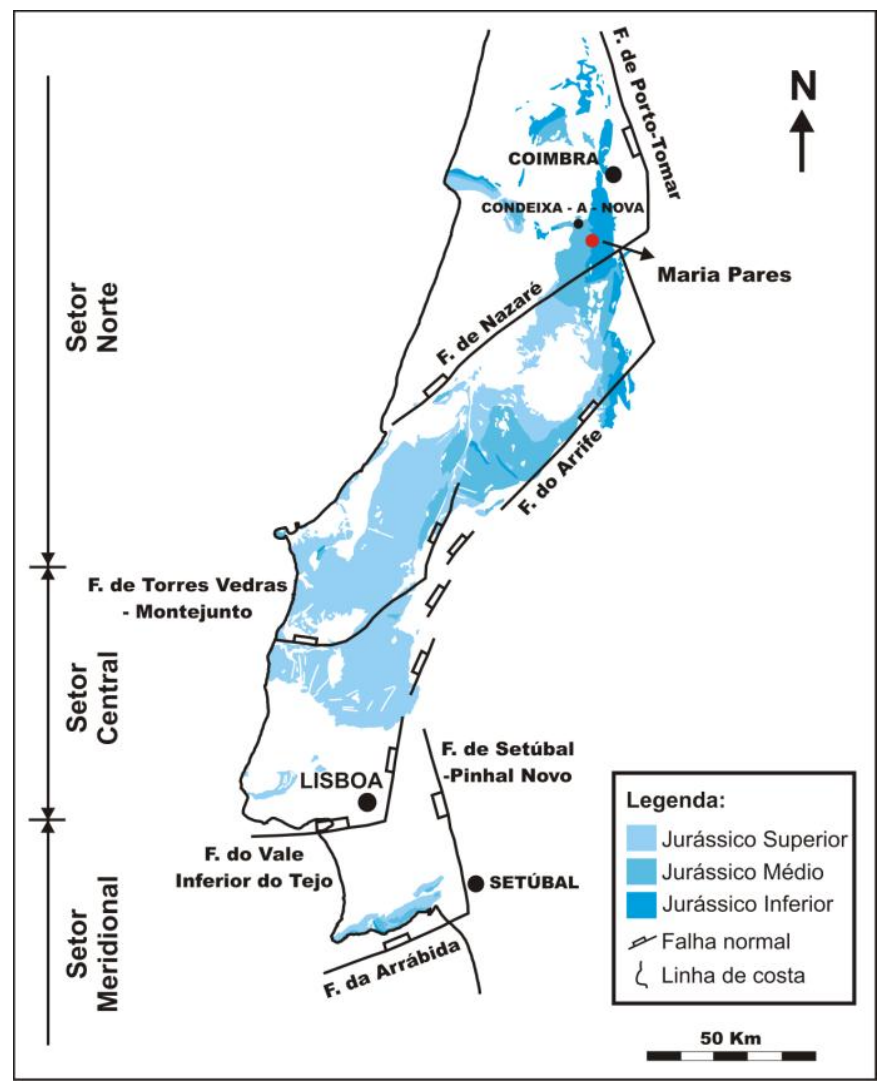

Fig. 1 - Localização do perfil de Maria Pares (Setor Norte da Bacia Lusitânica) (Modificado da CARTA GEOLÓGICA DE PORTUGAL, 1992 e adaptado de KULLBERG, 2000). 
Do ponto de vista litostratigráfico, o intervalo estudado (Toarciano superior - Aaleniano inferior - Aaleniano médio) corresponde maioritariamente à Formação de Póvoa da Lomba (sensu AZERÊDO et al., 2003; DUARTE, 2007; BARBOSA et al., 2008), caraterizada pela alternância de camadas de margas e calcários margosos de cor cinzenta, muito ricos em amonites, com predominância de margas na base e um aumento progressivo de calcário margoso para o topo (Fig. 2). Sobre esta unidade assenta a Formação de Degracias (AZERÊDO et al., 2003), equivalente à Formação de Póvoa da Lomba para BARBOSA et al. (2008), que se carateriza pela ocorrência de calcários micríticos compactos em bancadas espessas, de cor cinzento-azulado (Calcários azuis do Poço Central em HENRIQUES, 1992). Entre elas, a ausência de registo da Biozona Murchisonae, com valor bacinal (HENRIQUES, 1992, 1998; AZERÊDO et al., 2003) traduz-se numa lacuna estratigráfica com expressão à escala regional (descontinuidade DT5 de DUARTE, 1997).

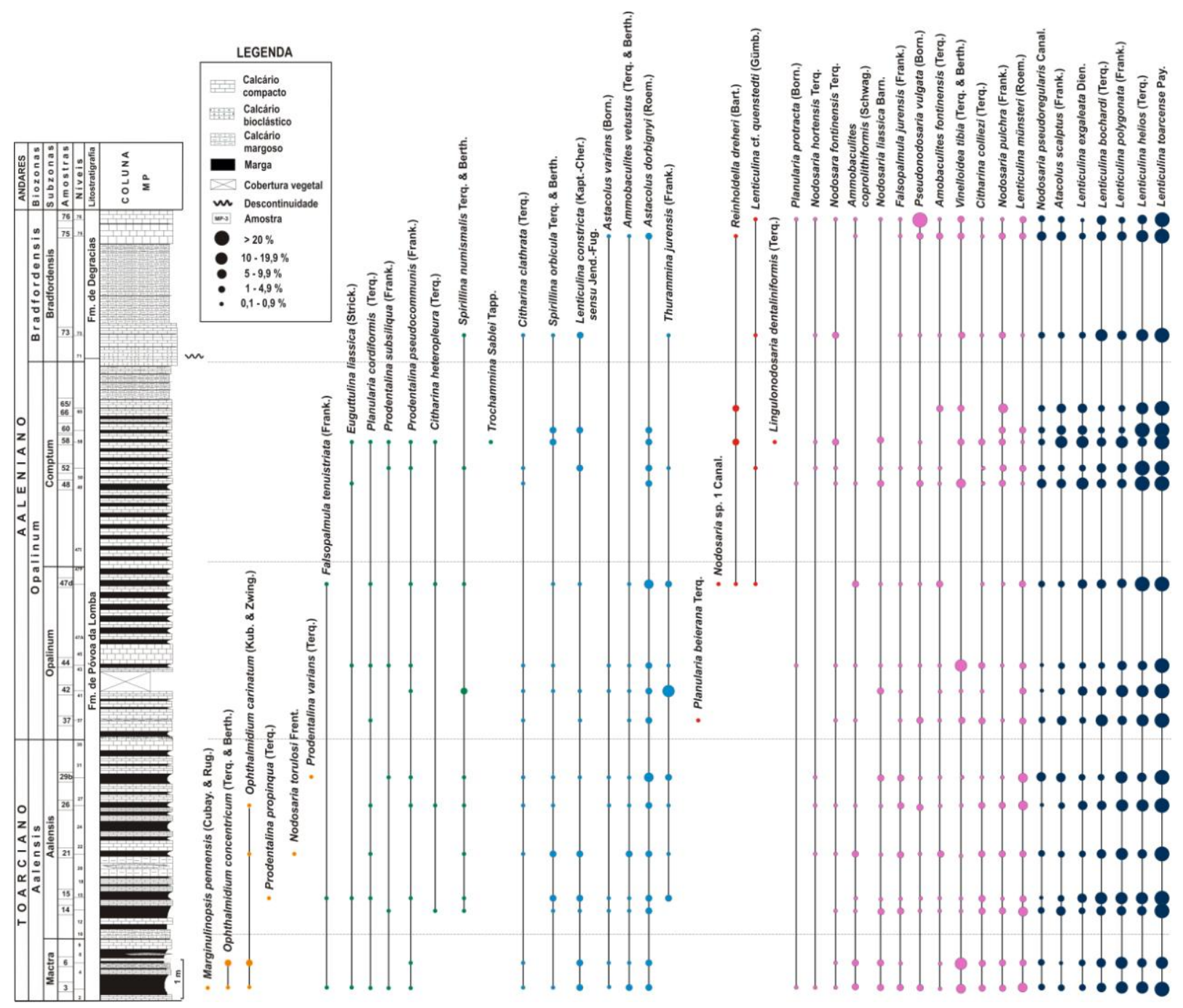

Fig. 2 - Perfil estratigráfico de Maria Pares e respetivas unidades biostratigráficas (HENRIQUES, 1992, 1998). Posicionamento das amostras estudadas ao longo do intervalo estratigráfico Toarciano superior - Aaleniano médio. Representação das ocorrências e abundância relativa de cada espécie identificada em cada associação de foraminíferos (GUTERRES, 2010). 


\section{3 - MATERIAIS E MÉTODOS}

A amostragem no perfil estudado realizou-se tendo em conta as unidades biostratigráficas estabelecidas por HENRIQUES (1992, 1998), através do reconhecimento de associações de amonites. Foram recolhidas, no total, 19 amostras (Fig. 2), tendo sempre em atenção a espessura e as características litológicas das diferentes subzonas.

No trabalho de laboratório processaram-se cerca de 300 gr de cada amostra. Na lavagem das amostras utilizou-se uma coluna de 5 peneiros, com as malhas de $1 \mathrm{~mm}, 0,5 \mathrm{~mm}$, 0,250mm, 0,125mm e 0,063mm. Depois da triagem das três frações intermédias, a classificação dos exemplares, a nível supragenérico, baseou-se na classificação de LOEBLICH e TAPPAN (1988). A nível específico, utilizou-se a base de dados de descrições originais elaborada por ELLIS e MESSINA (1940-1990).

$\mathrm{Na}$ abordagem paleoecológica das associações de foraminíferos registadas no perfil estudado, foram utilizados alguns índices de diversidade que se podem agrupar em dois tipos: índices de riqueza específica e índices baseados na dominância de espécies (CANALES, 1998, 2001). Quanto aos índices de riqueza, aplicaram-se o Índice $\alpha$ de Fisher e o Índice de Riqueza de Margalef. Dos índices baseados na dominância de espécies, usaram-se o Índice de Simpson, o Índice de Berger - Parker, o Índice de Shannon e Wiener e o Índice de Equitabilidade de Pielou. Os valores de tais índices nas associações estudadas foram obtidos utilizando o programa PAST (HAMMER e HARPER, 2006). As variações destes valores obtidos, ao longo do intervalo estratigráfico estudado, foram posteriormente analisadas e interpretadas.

\section{4 - RESULTADOS E DISCUSSÃO}

Nas associações estudadas obtiveram-se, no total, 15.273 foraminíferos que se incluem em 5 subordens, 12 famílias, 18 géneros e 45 espécies (GUTERRES, 2010; CANALES et al., 2010) (Fig. 2). A composição taxonómica das associações estudadas corresponde ao domínio boreal e são típicas de plataformas carbonatadas do Jurássico (HALLAM, 1969; GORDON, 1970; CANALES, 1998, 2001). Os exemplares apresentam-se relativamente bem preservados e não se encontra nenhuma evidência que indique que a composição das associações tivesse sido afetada por mecanismos de alteração tafonómica.

Em relação aos índices aplicados, verificou-se que o Índice $\alpha$ de Fisher nas associações estudadas varia entre 3,53 e 6,76 (Fig. 3A), com valores relativamente regulares e altos $(\alpha>$ 5) em grande parte das amostras (12), o que traduz ambientes marinhos normais (MURRAY, 1991), com condições ambientais adequadas ao desenvolvimento das associações de foraminíferos. Ao longo de todo o perfil verifica-se uma certa homogeneidade dos valores deste índice, mas na Biozona Bradfordensis os valores baixam $(\alpha<4)$, o que pode refletir mudanças ambientais inibidoras do normal desenvolvimento de algumas associações (MURRAY, 1991).

Nas associações estudadas, os valores do Índice de Riqueza de Margalef variam entre 2,46 e 4,72 (Fig. 3B). Verifica-se que existe uma certa homogeneidade dos valores (entre 3 e 4) em quase todo o perfil, à exceção do topo da Subzona Comptum, em que se regista uma pequena oscilação, refletindo condições ambientais um pouco instáveis.

O Índice de Simpson varia entre 0 e 1 e reflete a dominância numa associação. Se todos os indivíduos são da mesma espécie, $\lambda$ é 1 . Isto significa que, quanto maior é $\lambda$, menor é a diversidade (BUZAS, 1979). Os valores obtidos para este índice variam entre 0,69 e 0,91 nas diferentes associações (Fig. 3C). Na sua maioria, os valores tendem a ser altos, $\operatorname{com} \lambda>0,8$. Somente em duas associações (MP65/66 e MP73) é que o valor é inferior a 0,7. Estes valores indicam que, apesar de se registarem valores altos de diversidade nas associações estudadas, 
há um certo domínio de uma espécie que, em todas as associações é Lenticulina toarcense Payard.

O Índice de Berger - Parker permite quantificar a dominância. Os valores obtidos para este índice, nas associações estudadas, mostram variações entre 0,20 e 0,53 (Fig. 3D). Estes valores são, no geral, baixos e apresentam oscilações pouco significativas. Os valores deste índice reforçam que nas associações estudadas, a dominância de Lenticulina toarcense Payard é moderada.

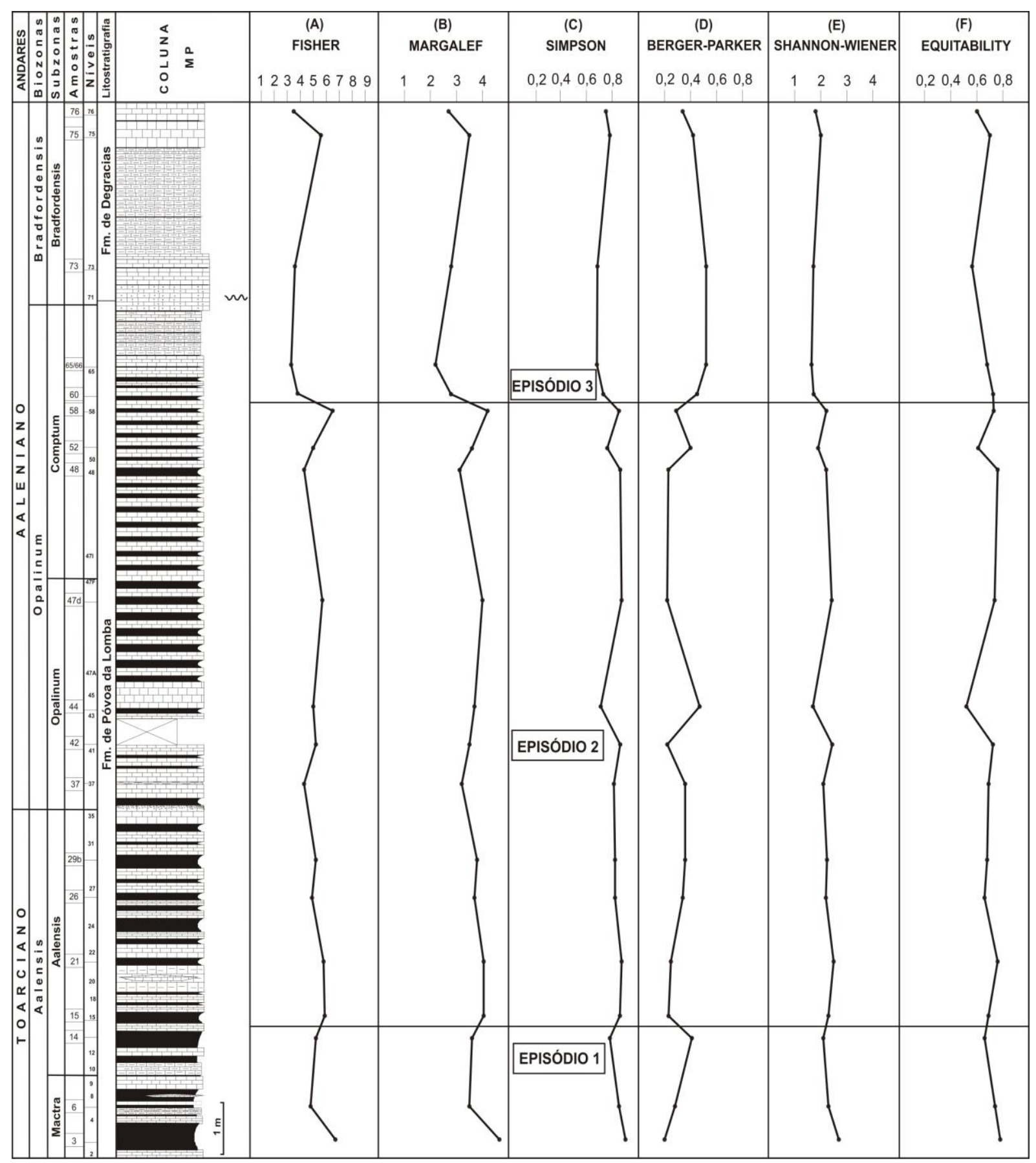

Fig. 3 - Representação gráfica dos valores dos índices de diversidade aplicados e episódios separados a partir das suas variações. (A) Índice $\alpha$ de Fisher; (B) Índice de Riqueza de Margalef; (C) Índice de Simpson; (D) Índice de Berger - Parker; (E) Índice de Shannon e Wiener; (F) Índice de equitabilidade de Pielou. 
Os valores do Índice de Shannon e Wiener no Perfil de Maria Pares permanecem relativamente constantes ao longo de todo o intervalo estratigráfico estudado, variando entre 1,71 e 2,76 (Fig. 3E). BEERBOWER e JORDAN (1969) consideraram que a diversidade é baixa quando $H^{\prime}<0,6$, moderada quando $0,6<\mathrm{H}^{\prime}<1$ e alta quando $\mathrm{H}^{\prime}>1$. Assim, no Perfil de Maria Pares, pode considerar-se que as associações de foraminíferos bentónicos são diversas. Por outro lado, MURRAY (1991) relaciona os valores da diversidade com distintos paleoambientes. Assim, segundo este autor, os valores obtidos com este índice indicam que as associações estudadas se desenvolveram em condições ambientais de plataforma marinha com salinidade normal.

O Índice de Equitabilidade de Pielou indica se as diferentes espécies constituintes de uma associação possuem abundância (número de exemplares) semelhante ou divergente. Assim, se $\mathrm{J}^{\prime}=1$, todas as espécies têm o mesmo número de exemplares, o que significa que não existe dominância de uma espécie em relação a outra. Se os valores forem baixos, tal indicador evidencia condições de elevado "stress" ambiental (HAYNES, 1981). No Perfil de Maria Pares, os valores do Índice de Equitabilidade de Pielou permanecem relativamente constantes, variando entre 0,53 e 0,79 (Fig. $3 \mathrm{~F}$ ). Assim sendo, não se deteta uma clara dominância de nenhuma espécie e, em momento algum, parece ter havido condições de "stress" ambiental.

A análise dos valores dos índices de diversidade obtidos no presente trabalho permite a diferenciação de três episódios ao longo do intervalo estratigráfico estudado (Fig. 3). Cada um destes episódios reflete condições ambientais ligeiramente diferentes, em termos de estabilidade ou instabilidade, que condicionaram a diversidade das associações.

No Episódio 1 (Subzona Mactra até à parte inferior da Subzona Aalensis), os índices mostram valores com tendência decrescente, que poderão indicar que as condições ambientais foram cada vez menos favoráveis ao desenvolvimento das associações de foraminíferos neste intervalo.

No Episódio 2 (Subzona Aalensis até à parte superior da Subzona Comptum), os valores dos índices são mais altos, estáveis e homogéneos, com exceção de pequenas oscilações no topo da Subzona Comptum. Os valores mais elevados e regulares traduzem condições ambientais estáveis e adequadas ao desenvolvimento das associações. Os valores mais oscilatórios, na parte superior deste episódio, podem refletir maior instabilidade nas condições ambientais, provavelmente relacionadas com mudança nas condições de sedimentação, de natureza cada vez mais calcária e menos margosa.

No Episódio 3 (Biozona Bradfordensis), os valores dos índices começam por ser baixos e apresentam uma tendência crescente. No entanto, os valores são mais baixos do que no Episódio 2, o que pode ser interpretado, em termos paleoecológicos, como uma perda inicial de qualidade nas condições ambientais, com tendência a melhorar na parte superior deste episódio. A instalação de condições de sedimentação mais calcárias, provavelmente não favorece o desenvolvimento de associações de foraminíferos tão abundantes e diversas como no Episódio 2.

\section{5 - CONCLUSÕES}

Do ponto de vista paleoecológico, as associações de foraminíferos registadas no Perfil de Maria Pares, ao longo do intervalo estratigráfico Toarciano superior - Aaleniano inferior Aaleniano médio, desenvolveram-se em zonas de plataforma marinha, com salinidade normal, bem oxigenadas e localizadas a profundidades situadas acima do nível de compensação da calcite. São constituídas por espécies com modos de vida bentónicos, tanto epifaunais como infaunais. As associações estudadas correspondem ao domínio boreal e são típicas de plataformas carbonatadas do Jurássico. 
A análise quantitativa das associações de foraminíferos, com o propósito de avaliar a sua diversidade, bem como a sua evolução ao longo do intervalo estratigráfico estudado, permite considerar que as associações analisadas são, em geral, abundantes e diversas e sem dominância de nenhuma espécie, apesar de Lenticulina toarcense Payard ser a espécie mais abundante em todas elas. A variação dos valores dos índices de diversidade aplicados permite a diferenciação de 3 episódios ao longo do intervalo estratigráfico estudado: o Episódio 1 (Subzona Mactra até à parte inferior da Subzona Aalensis) traduz condições ambientais cada vez menos favoráveis para o desenvolvimento das associações; o Episódio 2 (Subzona Aalensis até à parte superior da Subzona Comptum) traduz condições ambientais mais estáveis e adequadas ao desenvolvimento das associações. No entanto, em algumas associações registadas no topo da Subzona Comptum, verifica-se um decréscimo de exemplares e de espécies que, por sua vez, pode traduzir uma pequena instabilidade das condições ambientais; no Episódio 3 (Biozona Bradfordensis), as condições ambientais são inicialmente pouco favoráveis para o desenvolvimento das associações, mas com tendência a melhorar. É provável que o registo das associações de foraminíferos menos abundantes e diversas neste perfil, a partir do topo da Subzona Comptum, esteja relacionado com a mudança no regime de sedimentação, que passa a ser mais calcária e menos margosa do que no resto do intervalo estratigráfico estudado.

Agradecimentos - Os autores agradecem a María Luisa Canales (Universidade Complutense de Madrid, Espanha) e a Maria Helena Henriques (Universidade de Coimbra, Portugal) pela leitura crítica do manuscrito. O trabalho de laboratório beneficiou do apoio do Consórcio Petrobras-Galp-Partex.

\section{REFERÊNCIAS BIBLIOGRÁFICAS}

AZERÊDO A. C.; DUARTE, L. V.; HENRIQUES M. H. e MANUPPELlA, G. (2003) - Da dinâmica continental no Triásico aos mares do Jurássico Inferior e Médio. Cadernos de Geologia de Portugal, Instituto Geológico e Mineiro, Lisboa, 43 p.

BARBOSA, B.; SOARES, A. F.; ROCHA, R. B.; MANUPPELLA, G. e HENRIQUES, M. H. (2008) - Carta Geológica de Portugal, na escala 1:50.000. Notícia explicativa da Folha 19 - A (Cantanhede). Instituto Nacional de Engenharia, Tecnologia e Inovação, Lisboa, Portugal, 41 p.

BEERBOWER, J. R. e JORDAN, D. (1969) - Application of information theory to paleontologic problems: Taxonomic diversity. Journal of Paleontology, 43 (5), p. 1184-1198.

BUZAS, M. A. (1979) - The measurement of species diversity. Society of Economic Paleontologists and Mineralogists, Short Course, 6, p. 3-10.

CANALES, M. L. (1998) - Foraminíferos del Aaleniense en la Cuenca Vasco-Cantábrica. Tesis Doctoral. Departamento de Paleontología, Universidad Complutense de Madrid. 759 p. (inédita).

CANALES, M. L. (2001) - Los foraminíferos del Aaleniense (Jurásico Médio) en la Cuenca Vasco-Cantábrica (N de España). Revista Española de Micropaleontología, 33 (3), p. 253-438.

CANALES, M. L.; HENRIQUES, M. H.; FIGUEIREDO, V. L. e GUTERRES, H. (2010) - Foraminifera from the Lower-Middle Jurassic of the Lusitanian Basin (Portugal) - biostratigraphic and palaeoecological significance. In: Pena dos Reis, R. e Pimentel, N. (eds.). "Rediscovering the Atlantic: new ideas for an old sea...”, II Central \& North Atlantic Conjugate Margins Conference Lisbon, p. 42-46.

CARTA GEOLÓGICA DE PORTUGAL, à escala 1/500 000 (1992) - Serviços Geológicos de Portugal, Lisboa (2 Folhas).

DUARTE, L. V. (1997) - Facies analysis and sequential evolution of the Toarcian - Lower Aalenian series in the Lusitanian Basin (Portugal). Comunicação do Instituto Geológico e Minerio, Lisboa, 83, p. 65-94.

DUARTE, L. V. (2007) - Lithostratigraphy, sequence stratigraphy and depositional setting of the Pliensbachian and Toarcian series in the Lusitanian Basin, Portugal. Ciências da Terra, Universidade Nova, Lisboa, 16, p. 17-23, 5 figs.

ELLIS, B. F. e MESSINA, A. (1940-1990) - Catalogue of Foraminifera. Museum of Natural History, New York.

GORDON, W. A. (1970) - Biogeography of Jurassic Foraminifera. The Geological Society of America Bulletin, 81, p. 1689-1704. 
GUTERRES, H. C. (2010) - Foraminíferos da passagem Jurássico Inferior - Médio do Sector Norte da Bacia Lusitânica: o perfil de Maria Pares (Rabaçal). Tese de Mestrado, Universidade de Coimbra, 78 p. (não publicada).

HALLAM, A. (1969) - Faunal realms and facies in the Jurassic. Palaeontology, 12 (1), p. 1-18.

HAMMER, O. e HARPER, D. A. T. (2006) - Paleontological Data Analysis. Oxford, Blackwell Publishing, $351 \mathrm{p}$.

HAYNES, J. R. (1981) - Foraminifera. Macmillan Publishers Ltd., London, 433 p.

HENRIQUES, M. P. (1992) - Biostratigrafia e Paleontologia (Ammonoidea) do Aaleniano em Portugal (Sector Setentrional da Bacia Lusitaniana). Tese de Doutoramento. Centro de Geociências, Departamento de Ciências da Terra da Universidade de Coimbra, 301 p., 7 est. (não publicado).

HENRIQUES, M. P. (1998) - Caracterização biostratigráfica do Aaleniano de Maria Pares (Rabaçal, Portugal). In: Oliveira, J. T. e Dias, R. P. (eds.): V Congresso Nacional de Geologia, Livro Guia das Excursões, Lisboa. Sociedade Geológica de Portugal, 1, p. 53-57.

KULlBERG, J. C. (2000) - Evolução Tectónica Mesozóica da Bacia Lusitaniana. Tese de Doutoramento em Geologia, Faculdade de Ciências e Tecnologia, Universidade Nova de Lisboa, 280 p. (não publicada).

LOEBLICH, A. R. e TAPPAN, H. (1988) - Foraminifera genera and their classification. Van Nostrand Reinhold Company, New York, vol. 2, 970 p.

MURRAY, J. W. (1991) - Ecology and Paleoecology of Benthic Foraminifera. John Wiley and Sons Inc., New York, 397 p. 